\title{
Ion conducting properties of imidazolium salts with tri-alkyl chains in organic electrolytes against activated carbon electrodes
}

\author{
Kyungmin Kim ${ }^{1}$, Soo-Jin Park ${ }^{2}$, Ji Sun $\mathrm{Im}^{3}$, Chul Wee Lee ${ }^{3}$, Yongju Jung ${ }^{4}$ and Seok Kim ${ }^{1, \star}$ \\ ${ }^{1}$ School of Chemical and Biochemical Engineering, Pusan National University, Busan 46241, Korea \\ ${ }^{2}$ Department of Chemistry, Inha University, Incheon 22212, Korea \\ ${ }^{3}$ C-Industry Incubation Center, Korea Research Institute of Chemical Technology (KRICT), Daejeon 34114, Korea \\ ${ }^{4}$ Department of Applied Chemical Engineering, Korea University of Technology and Education, Cheonan 31253, Korea
}

Key words: imidazolium, ionic liquids, organic electrolyte, conducting property, capacitors

\begin{abstract}
Article Info
Received 14 July 2015

Accepted 6 September 2015

*Corresponding Author

E-mail: seokkim@pusan.ac.kr

Tel: $+82-51-510-3874$

Open Access

DOI: http://dx.doi.org/

10.5714/CL.2016.17.1.070

This is an Open Access article distributed under the terms of the Creative Commons Attribution Non-Commercial License (http://creativecommons.org/licenses/ by-nc/3.0/) which permits unrestricted non-commercial use, distribution, and reproduction in any medium, provided the original work is properly cited.
\end{abstract}

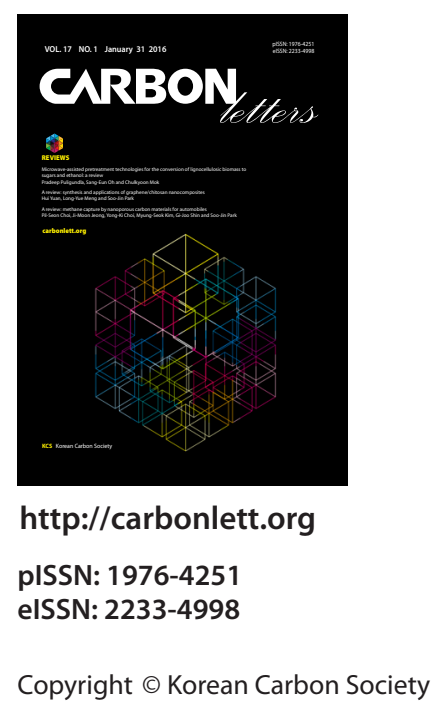

Electrochemical double layer capacitors (EDLCs), a type of energy storage device, are currently receiving considerable attention. They have a high power density and good cycle ability. Furthermore, they operate on a simple mechanism where electrical charges in an electrochemical double layer are accumulated at the interface between the electrode and the electrolyte [1-3]. For these reasons, capacitors are used in a wide range of applications such as mobile phones, electrical vehicles, and industry power supplies.

Capacitors generally consist of an electrode and an electrolyte. The electrode is prepared using carbon materials such as activated carbon, graphene, or graphite fibers [4-7]. Among the carbon materials, activated carbon, which has a high specific surface area and a large number of pores, is suitable for the capacitor electrode. In particular, it readily absorbs or desorbs electric charge. The electrolyte, meanwhile, can be divided into aqueous and nonaqueous electrolytes. Non-aqueous electrolytes have a wide electrochemical stability of operative voltage compare to aqueous electrolytes. Because the window potential is related to the energy density $\left(E=1 / 2 \mathrm{~V}^{2}\right)$, the voltage limit of the electrolyte is an important factor for the characteristics of a capacitor [2,8-10]. The ionic conductivity, capacitance, and impedance of electrochemical stability are also significant parameters of the electrolyte.

Recently, ionic liquids (IL), which are also known as room temperature molten salts, have attracted attention for use in electrolytes due to their unique characteristics such as non-flammability, high ionic conductivity, low melting points, and wide electrochemical stability window. ILs have large cations and organic or inorganic anions in a liquid state at room temperature [11-15].

Among various ILs, imidazolium based ILs are used most widely because the hydrogen of imidazolium can be easily substituted. In particular, imidazolium, which has 1-methyl3-butyl side chains, is widely used in many fields of study due to its relatively low viscosity, high conductivity, and low melting point. A capacitor using 10\% 1-butyl-3-methyl imidazolium tetrafluoroborate $\left(\mathrm{BMImBF}_{4}\right)$ was found to have the best electrochemical performance in our previous study [16]. Although imidazolium cation with 1,2,3-alkyl side chains has high viscosity, it presents excellent electrochemical stability [17]. However, the influence of 1,2,3-alkyl substituted imidazolium salts on the ion conducting property of organic electrolytes is not fully understood.

The objective of this study was to find the optimal proportion of 1-butyl-2,3-dimethyl imidazolium tetrafluoroborate $\left(\mathrm{BMMImBF}_{4}\right)$, which is a tri-alkyl substituted imidazolium salt, as an additive in an organic electrolyte and to confirm the electrochemical performance of the resultant electrolyte for capacitor applications. $\mathrm{BMMImBF}_{4}$ is an attractive candidate as an additive in organic electrolytes of EDLCs.

The electrodes were composed of the activated materials, conductive agents, binders, and substrate. Activated carbon (MSP-20; Kuraray Chemical, Osaka, Japan) and carbon black (Super P; Alfa Aesar, Ward Hill, MA, USA) are used as an activated material and 


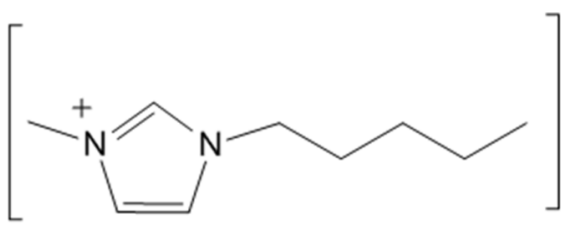

$\mathrm{BF}_{4}$

(a)

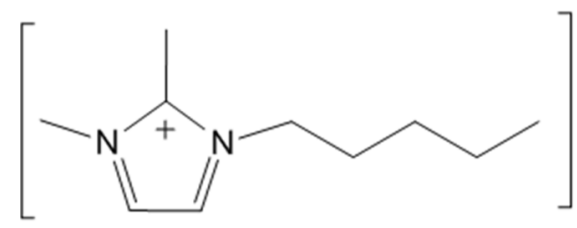

$\mathrm{BF}_{4}$

(b)

Fig. 1. The structure of (a) 1-butyl-3-methyl imidazolium tetrafluoroborate $\left(\mathrm{BMImBF}_{4}\right)$ and (b) 1-butyl-2,3-dimethyl imidazolium tetrafluoroborate $\left(\mathrm{BMMImBF}_{4}\right)$.

conductive agent, respectively. Carboxymethylcellulose (CMC; Aldrich, St. Louis, MO, USA) / styrene butadiene rubber (SBR) serves as a binder. These materials are well mixed with N-methylpyrrolidone (NMP; Junsei Chemical, Tokyo, Japan) using agate mortar for 30 minutes in a glove box. The mixed slurry (activated carbon : carbon blacks : $\mathrm{CMC}: \mathrm{SBR}=85: 5$ : $6: 4[\mathrm{wt} \%])$ was then coated on a Ni foam substrate $(1 \mathrm{~cm} \times 1$ $\mathrm{cm})$ and dried at $100^{\circ} \mathrm{C}$ in a vacuum oven for $12 \mathrm{~h}$. This process is carried out to remove and evaporate the NMP. The prepared electrodes were pressed. The average loading weight of the active materials was about $10 \mathrm{mg} / \mathrm{cm}^{2}$. The final film electrode was applied to an ELDC.

The electrolytes were prepared using $0.1 \mathrm{M}$ tetraethyl ammonium tetrafluoroborate $\left(\mathrm{TEABF}_{4}\right.$; Aldrich) salts dissolved in ethylene carbonate (EC; Aldrich) and dimethoxyethane (DME; Daejung, Busan, Korea) $(5 / 5$ vol\%) in a glove box. Different volume ratios $(5,10,15$, and $20 \mathrm{vol} \%)$ of $\mathrm{BMMImBF}_{4}$ were added to the prepared electrolyte, which was then stirred for 12 h. The chemical structures of $\mathrm{BMImBF}_{4}$ and $\mathrm{BMMImBF}_{4}$ are shown in Fig. 1. These final organic electrolytes solutions were used as electrolytes for EDLCs.

The ionic conductivities of the electrolytes were examined using $\mathrm{AC}$ impedance spectroscopy at a frequency range from $1000 \mathrm{kHz}$ to $1 \mathrm{~Hz}$ at $0.1 \mathrm{~V}$ amplitude at room temperature.

The electrochemical stability of the electrolytes was confirmed by linear sweep voltammetry at a scan rate of $10 \mathrm{mV} \cdot \mathrm{s}^{-1}$. Pt wires served as counter electrodes and an $\mathrm{Ag} / \mathrm{Ag}^{+}$electrode was used as a reference electrode.

The thermal stability of the electrolyte with IL additives was measured using a themogravimetric analyzer (TGA). The mass of the electrolyte sample was varied about $20 \mathrm{mg}$. For the dynamic method, samples were heated from $30^{\circ} \mathrm{C}$ to $500^{\circ} \mathrm{C}$ at a heating rate of $10^{\circ} \mathrm{C} / \mathrm{min}$.

The characterizations of the electrochemical perfomance including the capacitance, operative voltage, resistance, and cycle ability were carried out using cyclic voltammetry (CV) and electrochemical impedance spectroscopy (EIS). The evaluations were carried out in a three electrode system using an Iviumstat (Ivium Technologies, Eindhoven, the Netherlands).

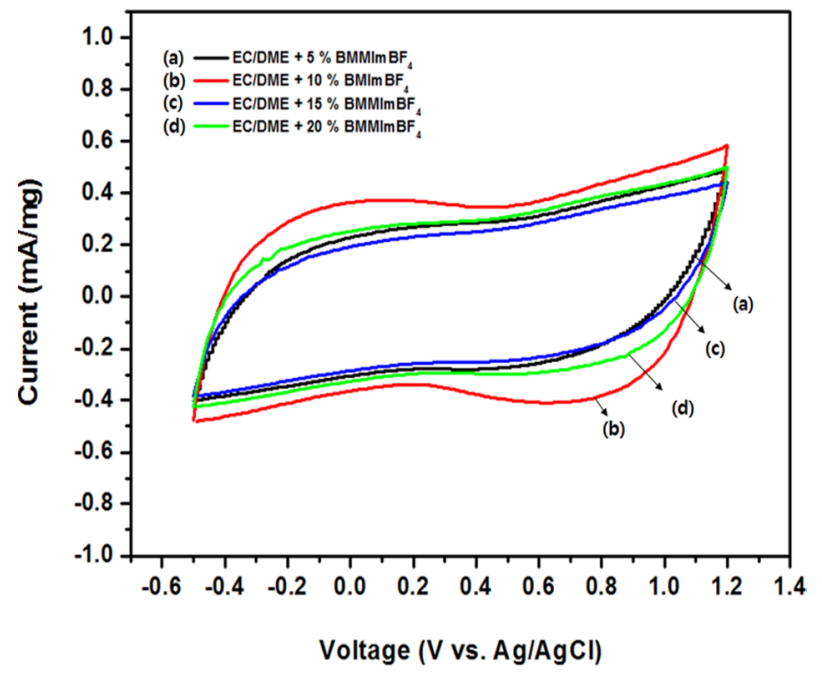

Fig. 2. Cyclic voltammograms of capacitor containing EC/DME electrolyte with $0.1 \mathrm{M}$ tetraethyl ammonium tetrafluoroborate $\left(\mathrm{TEABF}_{4}\right)$ with different ratios of BMMImBF 4 at a scan rate of $5 \mathrm{mV} \cdot \mathrm{s}^{-1}$. EC, ethylene carbonate; $\mathrm{DME}$, dimethoxyethane; $\mathrm{BMMImBF}_{4}$, 1-butyl-2,3-dimethyl imidazolium tetrafluoroborate; $\mathrm{BMImBF}_{4}, 1$-butyl-3-methyl imidazolium tetrafluoroborate.

The working electrode was the prepared electode. $\mathrm{AnAg} / \mathrm{AgCl}$ electrode and platinum wire were used as reference and counter electrodes, respectively.

The EIS was performed by AC impedance spetroscopy over a frequency range from $100 \mathrm{kHz}$ to $0.01 \mathrm{~Hz}$ with $5 \mathrm{mV}$ amplitude modulation.

The ion conducting property of an electrolyte for capacitor applications is one of the most important factors for the capacitive behavior of the capacitor. Among the various properties, the ionic conductivity of a solution has a critical effect on the electrochemical performance of the capacitor.

The variation of the ionic conductivity of the $\mathrm{BMMImBF}_{4^{-}}$ organic solvent mixture per volume percent of ILs is presented in Table 1. From the conductivity results, as more IL was added, the ionic conductivity increased at room temperature. It appears that $\mathrm{BMMImBF}_{4}$ as an additive can ion charges effectively migrate or diffuse.

The CVs of EDLCs using the $\mathrm{BMMImBF}_{4}$ additive in an organic electrolyte at a scan rate of $5 \mathrm{mV} \cdot \mathrm{s}^{-1}$ demonstrate the electrochemical performance, as presented in Fig. 2. Rectangular shapes were observed, corresponding with a typical EDLC. The specific capacitance (C) of EDLCs is defined by the following equation (1):

$$
C=\frac{\int I d V}{v m \Delta V}
$$

$\mathrm{I}$ and $\mathrm{V}$ denote the response current density $\left(\mathrm{Acm}^{-2}\right)$ and the scan rate of potential $\left(\mathrm{V} \cdot \mathrm{s}^{-1}\right)$, respectively. $\mathrm{m}$ is the active material weight of the working electrode $(\mathrm{g})$ and $\Delta \mathrm{V}$ is the operative voltage $(\mathrm{V})$ in a 3 electrode system $[18,19]$.

The specific capacitances were calculated and are presented in Table 1 . The capacitances increased to $133.7 \mathrm{~F} \cdot \mathrm{g}^{-1}$ with addition of $\mathrm{BMMImBF}_{4}$ ILs additives at $10 \%$. It is speculated that ions were effectively adsorbed and desorbed between the electrolyte and the surface or pores of the electrode. However, the 
Table 1. Resistances, conductivity, and capacitance of organic electrolytes containing ionic liquid additives

\begin{tabular}{|c|c|c|c|c|c|c|}
\hline \multirow{2}{*}{$\mathrm{BMMImBF}_{4}(\%)$} & \multicolumn{4}{|c|}{ Resistance $(\Omega)$} & \multirow{2}{*}{ Conductivity $\left(\mathrm{mScm}^{-1}\right)$} & \multirow{2}{*}{ Capacitance $\left(\mathrm{F} \cdot \mathrm{g}^{-1}\right)$} \\
\hline & $\mathrm{R}_{1}$ & $\mathrm{R}_{2}$ & $\mathrm{R}_{3}$ & W & & \\
\hline 5 & 21.6 & 42.3 & 18.9 & 12.8 & 4.1 & 94.7 \\
\hline 10 & 11.9 & 11.5 & 7.6 & 5.1 & 5.6 & 133.7 \\
\hline 15 & 12.9 & 24.1 & 12.0 & 9.9 & 6.3 & 95.9 \\
\hline 20 & 35.2 & 34.9 & 24.6 & 8.5 & 6.9 & 107.6 \\
\hline
\end{tabular}

$\mathrm{BMMImBF}_{4}$, 1-butyl-2,3-dimethyl imidazolium tetrafluoroborate; $R_{1}$, bulk resistance; $R_{2}$, deposited film resistance; $R_{3}$, charge transfer resistance; $W$, Warburg resistance.

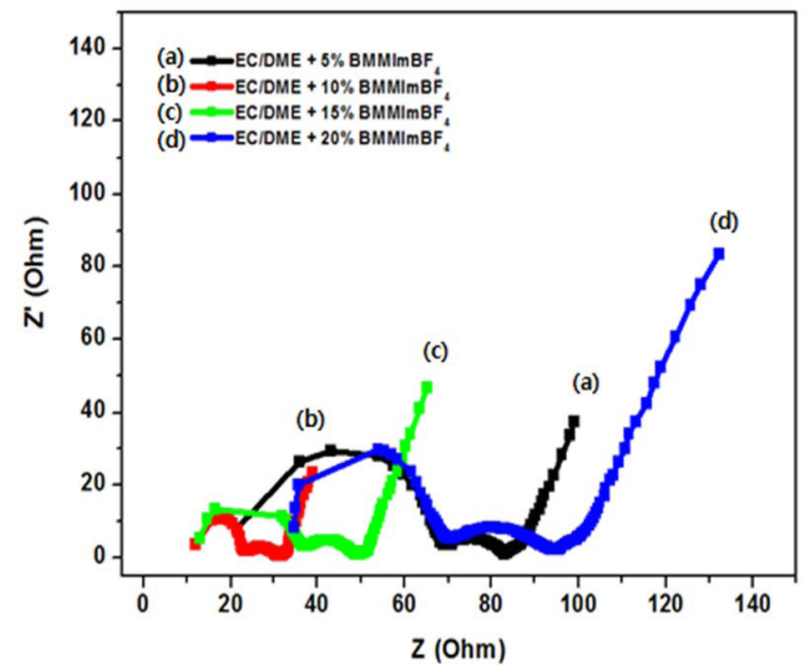

Fig. 3. Impedance spectra according to content of $\mathrm{BMMImBF}_{4}$ ionic liquids in organic electrolyte. $\mathrm{EC}$, ethylene carbonate; $\mathrm{DME}$, dimethoxyethane; $\mathrm{BMMImBF}_{4}$, 1-butyl-2,3-dimethyl imidazolium tetrafluoroborate.

capacitances decreased when the amount of additive exceeded $10 \%$. It appears that too many ion charges of ILs were saturated and interrupted the movement of ion charges, thereby having adverse effects on the interface between the electrode and the electrolyte.

$\mathrm{AC}$ impedance spectra of all electrolytes are presented in Fig. 3 , which shows two circles and vertical lines. The initial part of the plot and the diameter of the semi-circle indicate the bulk resistance $\left(R_{1}\right)$ and the deposited film resistance $\left(R_{2}\right)$, respectively, at high frequency. The diameter of the second semi-circle signifies the charge transfer resistance $\left(\mathrm{R}_{3}\right)$ at intermediate frequency. The Warburg resistance (W), which presents a straight line with a slope of $45^{\circ}$, defines the ion diffusion in an activated carbon electrode. The character of a pure EDLC could be confirmed by bulk impedance at low frequency [20]. The estimated values of resistances are indicated in Table.1. As shown in Fig. 3, the EDLCs containing $10 \% \mathrm{BMMImBF}_{4}$ in the organic electrolyte displayed the lowest charge transfer resistance and Warburg resistance. The results of this test indicated that the use of $\mathrm{BMMImBF}_{4}$ has a positive effect of increasing the capacitance of the EDLC.

Fig. 4 shows the capacitance retention behavior at prolonged charge-discharge cycles. It was evaluated in a range of $-0.5 \mathrm{~V}$ to $1.2 \mathrm{~V}$ for 1000 cycles at $100 \mathrm{mV} \cdot \mathrm{s}^{-1}$. The cycle stability is a

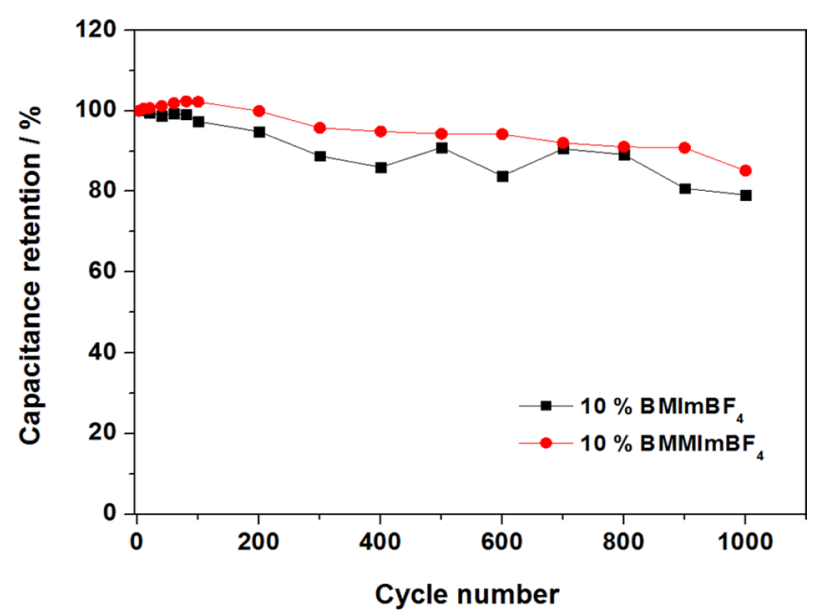

Fig. 4. Capacitance retention of electrochemical double layer capacitor containing $10 \%$ imidazolium ionic liquids with di-alkyl and tri-alkyl chains at a scan rate of $100 \mathrm{mV} \cdot \mathrm{s}^{-1}$. $\mathrm{BMImBF}_{4}, 1$-butyl-3-methyl imidazolium tetrafluoroborate; $\mathrm{BMMImBF}_{4}$, 1-butyl-2,3-dimethyl imidazolium tetrafluoroborate.

very important parameter to evaluate capacitor performance. As shown in Fig. 4, the cycle retention using $10 \% \mathrm{BMMImBF}_{4}$ in the electrolyte decreased by only $15 \%$, whereas a $20 \%$ decrease was observed in the case of using the $10 \%$ BMImBF4 additives. It is thought that the 1,2,3-tri-alkyl imidazolium cation is more stable than the 1,2-dialkyl imidazolium cation, because the disubstituted- imidazolium cation has low cathodic stability in terms of electrochemical performance and it undergoes some side reactions such as cathodic dimerization or di-alkylation reactions including the acidic proton in the two positions in Fig. 1. As a result, 1,2,3-tri-alkyl imidazolium cation based-ILs exhibited outstanding cycle ability, compared with the 1,2-dialkyl imidazolium ILs [21,22]. This is likely related with the tri-alkyl imidazolium cations having larger volume size than di-alkyl imidazolium cations. This cation volume size difference will affect the ion dissociation and the charge accumulation onto the activated carbons surfaces. By using molecular modification techniques, we could alter the ion mobility or charge capacitance, thereby affecting the electrochemical performance of the capacitor.

In this study, the effect of imidazolium cations with tri-alkyl chain substitutions in the organic electrolyte of a capacitor was investigated by chemical and electrochemical characterization. 
Reduced resistance and enhanced capacitance of $133.7 \mathrm{~F} \cdot \mathrm{g}^{-1}$ indicated that the $\mathrm{BMMImBF}_{4}$ IL could function as an effective additive in the capacitor electrolyte. In particular, the capacitor containing a small amount $(10 \%)$ of $\mathrm{BMMImBF}_{4}$ showed the maximum capacitance and low resistance at the interface between the electrode and the electrolyte resulting from the effective electric double layer thickness. Also, the cycle stability ( $85 \%$ retention after 1000 cycles) of the capacitor using tri-alkyl based imidazolium ILs was superior to that of the capacitor using di-alkyl based imidazolium. It could be concluded that the improved capacitance behaviors were dependent on the ion mobility and on the charge accumulation onto the surface of the activated carbon electrode by changing the molecular structure of additive salts. In conclusion, the $\mathrm{BMMImBF}_{4} \mathrm{IL}$ is a promising additive for organic electrolytes for capacitor and other electrochemical applications.

\section{Conflict of Interest}

No potential conflict of interest relevant to this article was reported.

\section{Acknowledgements}

This research was partly supported by the Basic Science Research Program through the National Research Foundation of Korea (NRF) funded by the Ministry of Science, ICT and Future Planning, Korea (Grant No.: NRF-2011-0009007).

\section{References}

[1] Gao ZH, Zhang H, Cao GP, Han MF, Yang YS. Spherical porous $\mathrm{VN}$ and NiOx as electrode materials for asymmetric supercapacitor. Electrochim Acta, 87, 375 (2013). http://dx.doi.org/10.1016/j. electacta.2012.09.075.

[2] Anouti M, Couadou E, Timperman L, Galiano H. Protic ionic liquid as electrolyte for high-densities electrochemical double layer capacitors with activated carbon electrode material. Electrochim Acta, 64, 110 (2012). http://dx.doi.org/10.1016/j.electacta.2011.12.120.

[3] Oh M, Kim S. Synthesis and electrochemical analysis of polyaniline $/ \mathrm{TiO}_{2}$ composites prepared with various molar ratios between aniline monomer and para-toluenesulfonic acid. Electrochim Acta, 78, 279 (2012). http://dx.doi.org/10.1016/j.electacta.2012.05.109.

[4] Kim MG, Kim K, Kim S. Conducting and interface characterization of carbonate-type organic electrolytes containing EMImBF4 as an additive against activated carbon electrode, Carbon Letters, 16, 51 (2015) DOI: http://dx.doi.org/10.5714/CL.2015.16.1.051

[5] Lota G, Grzyb B, Machnikowska H, Machnikowski J, Frackowiak E. Effect of nitrogen in carbon electrode on the supercapacitor performance. Chem Phys Lett, 404, 53 (2005). http://dx.doi. org/10.1016/j.cplett.2005.01.074.

[6] Kim K, Jung Y, Kim S. Influence of ionic liquid additives on the conducting and interfacial properties of organic solvent-based electrolytes against an activated carbon electrode, Carbon Letters, 15, 187 (2014) DOI: http://dx.doi.org/10.5714/CL.2014.15.3.187.
[7] Oh M, Park SJ, Jung Y, Kim S. Electrochemical properties of polyaniline composite electrodes prepared by in-situ polymerization in titanium dioxide dispersed aqueous solution. Synth Met, 162, 695 (2012). http://dx.doi.org/10.1016/j.synthmet.2012.02.021.

[8] Kim M, Kim S. Electrochemical properties of non-aqueous electrolytes containing spiro-type ammonium salts. J Ind Eng Chem, 20, 4447 (2014). http://dx.doi.org/10.1016/j.jiec.2014.02.015.

[9] Burke A. R\&D considerations for the performance and application of electrochemical capacitors. Electrochim Acta, 53, 1083 (2007). http://dx.doi.org/10.1016/j.electacta.2007.01.011.

[10] Kim M, Kim IJ, Yang S, Kim S. Fluoroethylene carbonate addition effect on electrochemical properties of mixed carbonate-based organic electrolyte solution for a capacitor. Bull Korean Chem Soc, 35, 466 (2014). http://dx.doi.org/10.5012/bkcs.2014.35.2.466.

[11] Wilkes JS, Zaworotko MJ. Air and water stable 1-ethyl-3-methylimidazolium based ionic liquids. J Chem Soc Chem Commun, (13), 965 (1992). http://dx.doi.org/10.1039/C39920000965.

[12] Paul A, Mandal PK, Samanta A. How transparent are the imidazolium ionic liquids? A case study with 1-methyl-3-butylimidazolium hexafluorophosphate, [bmim][PF6]. Chem Phys Lett, 402, 375 (2005). http://dx.doi.org/10.1016/j.cplett.2004.12.060.

[13] Bonhôte P, Dias AP, Papageorgiou N, Kalyanasundaram K, Grätzel M. Hydrophobic, highly conductive ambient-temperature molten salts. Inorg Chem, 35, 1168 (1996). http://dx.doi.org/10.1021/ ic951325x.

[14] Brandt A, Balducci A. Theoretical and practical energy limitations of organic and ionic liquid-based electrolytes for high voltage electrochemical double layer capacitors. J Power Sources, 250, 343 (2014). http://dx.doi.org/10.1016/j.jpowsour.2013.10.147.

[15] Welton T. Room-temperature ionic liquids: solvents for synthesis and catalysis. Chem Rev, 99, 2071 (1999). http://dx.doi. org/10.1021/cr980032t.

[16] Kim K, Kim S. Effect of alkyl-chain length of imidazolium based ionic liquid on ion conducting and interfacial properties of organic electrolytes. J Ind Eng Chem, 26, 136 (2015). http://dx.doi. org/10.1016/j.jiec.2014.11.025.

[17] Andriyko YO, Reischl W, Nauer GE. Trialkyl-substituted imidazolium-based ionic liquids for electrochemical applications: basic physicochemical properties. J Chem Eng Data, 54, 855 (2009). http://dx.doi.org/10.1021/je800636k.

[18] Yuan CZ, Gao BO, Zhang XG. Electrochemical capacitance of $\mathrm{NiO} / \mathrm{Ru}_{0.35} \mathrm{~V}_{0.65} \mathrm{O}_{2}$ asymmetric electrochemical capacitor. J Power Sources, 173, 606 (2007). http://dx.doi.org/10.1016/j.jpowsour.2007.04.034.

[19] Yu D, Dai L. Self-assembled graphene/carbon nanotube hybrid films for supercapacitors. J Phys Chem Lett, 1, 467 (2010). http:// dx.doi.org/10.1021/jz9003137.

[20] Kang J, Wen J, Jayaram SH, Yu A, Wang X. Development of an equivalent circuit model for electrochemical double layer capacitors (EDLCs) with distinct electrolytes. Electrochim Acta, 115, 587 (2014). http://dx.doi.org/10.1016/j.electacta.2013.11.002.

[21] Lane GH. Electrochemical reduction mechanisms and stabilities of some cation types used in ionic liquids and other organic salts. Electrochim Acta, 83, 513 (2012). http://dx.doi.org/10.1016/j.electacta.2012.08.046.

[22] Hunt PA. Why does a reduction in hydrogen bonding lead to an increase in viscosity for the 1-butyl-2,3-dimethyl-imidazoliumbased ionic liquids? J Phys Chem B, 111, 4844 (2007). http:// dx.doi.org/10.1021/jp067182p. 